Check for updates

Cite this: RSC Adv., 2017, 7, 50802

\title{
Strongly tricolor-emitting carbon dots synthesized by a combined aging-annealing route and their bio-application $\dagger$
}

\begin{abstract}
Ya Peng, (D) Xinyan Zhou, Nannan Zheng, Lingyu Wang and Xingping Zhou (D)*
Compared with the traditional semiconductor quantum dots and organic dyes, fluorescent carbon dots (FCDs) are superior because of their high hydrophilicity, robust chemical inertness, facile functionalization, high resistance to photobleaching, low toxicity, and good biocompatibility. However, to get FCDs with a high quantum yield (QY) in a large quantity has still been challenge recently. Here we report a novel and simple method, the combined aging-annealing way, by which the CA-N-FCDs with specific characters were prepared using citric acid (CA) and urea ( $\mathrm{N}$ source) as the raw materials. Compared with the use of a single hydrothermal treatment or annealing at relatively low temperature, the combination of the two methods can make excellent CDs. The QY of 78.8\% of the as-prepared CA-N-FCDs is one of the highest yields reported till now. The optimal conditions for preparing the CA-N-FCDs were obtained including the hydrothermal aging temperature, time and the annealing conditions. The aging time was $1.5 \mathrm{~h}$ at around $170{ }^{\circ} \mathrm{C}$, far lower than that used in the reported annealing or calcinating processes. The brightest sample was produced by annealing at $250{ }^{\circ} \mathrm{C}$ under the excitation of $365 \mathrm{~nm}$ light. The resulting uniform carbon nanoparticles with an average size of less than $10 \mathrm{~nm}$ were tricolor-emitting and blue was dominant, but including red and green colors. The CA-N-FCDs were of extremely low cell cytotoxicity applicable for being a kind of safe bio-material. Based on their high fluorescence quantum efficiency and the advantages mentioned above, these FCDs were then used for cell imaging and exhibited a perfect performance under 3 kinds of excitation bands (UV, blue, and green lights). Thus, these novel carbon dots will be able to be practically applied to immune labeling and imaging in vivo in the near future.
\end{abstract}

Received 21st September 2017 Accepted 25th October 2017

DOI: 10.1039/c7ra10471e

rsc.li/rsc-advances electrochemical oxidation. ${ }^{12}$ On the other hand, the "bottom-up" approaches synthesize CDs from molecular precursors such as ascorbic acid, ${ }^{13}$ glucose ${ }^{14}$ and polymer-silica nanocomposites ${ }^{15}$ through combustion, hydrothermal treatments/aging, calcination/annealing, and microwave synthetic routes. ${ }^{16}$ However, to get FCDs in a large scale with a high quantum yield (QY), with long fluorescence lifetime and high stability is still a challenge currently. Among these methods, the hydrothermal method $^{17}$ is more favored owing to its mild conditions such as easy operation and environmental friendliness. Meanwhile, calcination or annealing at relatively low temperature is also a very useful route due to its simplicity and high scale yield.

In order to rival organic dyes and semiconductor quantum dot counterparts, a high QY of CDs obtained under mind conditions with a large quantity is vital for those applications. ${ }^{18}$ In addition to surface-passivating, doping with heteroatoms such as nitrogen in particular has shown a great potential to significantly promote QY of the resulted CDs. ${ }^{19}$ But the majority of the CDs synthesized so far commonly have QY below $10 \%$, except that Wang et al. prepared CDs from oligomeric PEG diamine (PEG1500N) as surface passivation agent with a QY as high as $60 \%,{ }^{20}$ which puts them comparable on par with the best commercial CdSe/ZnS semiconductor quantum dots in
College of Chemistry, Chemical Engineering and Biotechnology, Donghua University, Shanghai 201620, China. E-mail: xpzhou@dhu.edu.cn

$\dagger$ Electronic supplementary information (ESI) available. See DOI: 10.1039/c7ra10471e 
aqueous solutions. Consequently, extensive research efforts have been devoted to engineering CDs in a large quantity to improve their QY, dispersity, and their stability.

Low cost and low cytotoxicity are two of the important determinants of CDs for being viable competitors to semiconductor quantum dots. However, many approaches require either precursors with high cost, complex instrumental set-ups or post-treatments to synthesize fluorescent CDs. If the hydrothermal treatment with a high scale yield and the annealing at relatively low temperature are combined, production of the excellent CDs can become desirable. Hereafter, the practical application will be pushed greatly.

To satisfy the requisites resulted from their real usages, we establish a novel way to make the product embrace industrial probability, with high quantum efficiency and high product quantity at the same time by the combined route consisting of a hydrothermal aging and the consequential annealing. In our approach, we select citric acid and urea as the precursory materials to make bright tricolor FCDs. On one hand, citric acid and urea, as cheap industrial raw materials, have been well received by people with a large solubility in water. On the other hand, urea contains a large number of nitrogen and oxygen atoms ${ }^{21}$ to ensure the generation of strong fluorescence by doping or co-doping. Thus, it becomes possible to get the CDs with a high QY and a low cost in a large scale. In this work, effects of the reaction conditions for the aging and annealing processes were investigated and some of their bio-applications were inspected for testing their possible practical application.

\section{Experimental section}

\subsection{Materials}

Citric acid monohydrate (CA), urea (N), anhydrous ethanol (99.7\%), filter membrane $(0.22 \mu \mathrm{m})$, Dulbecco modified eagle medium (DMEM), dimethyl sulphoxide (DMSO), penicillin, streptomycin, fetal bovine serum, phosphate buffered saline (PBS), 3-(4,5-dimethyl-2-thiazolyl)-2,5-diphenyl-2- $H$-tetrazolium bromide (MTT), and quinine sulfate were purchased from Sinopharm Chemical Reagent Co., Ltd., China. Qualitative filter was purchased from Hangzhou Special Paper Co., Ltd. Distilled water was prepared by our own lab. HeLa cells were purchased from the Chinese Academy of Sciences.

\subsection{Preparation of tricolor emission CA-N-FCDs}

A typical process for obtaining the CDs illustrated in Scheme 1 is also shown as the following. In $25 \mathrm{~mL}$ of the distilled water, $1.2 \mathrm{~g}$ citric acid and $0.8 \mathrm{~g}$ urea were mixed and dissolved thoroughly by ultrasonic treatment. Then, the mixture was transferred to a high pressure reactor and aged at $170{ }^{\circ} \mathrm{C}$ for $1.5 \mathrm{~h}$. After the product was fully dried, it was annealed for $0.5 \mathrm{~h}$ under $250{ }^{\circ} \mathrm{C}$ in the muffle furnace. Finally, the resultant was dispersed in the distilled water and the carbon dots suspension was obtained by the use of $0.22 \mu \mathrm{m}$ water filtration membrane. In order to achieve products with the stronger fluorescence in a large quantity, the investigation was carried out systematically under different hydrothermal conditions, including hydrothermal aging temperature, feeding ratio, maximum amount of feeding in an equal volume reaction system, aging time and annealing temperature.

\subsection{QY measurement}

Quantum yield of the sample was measured by using quinine sulfate as the standard substance. The QY of its $0.05 \mathrm{~mol} \mathrm{~L}^{-1}$ sulfuric acid solution is $54.0 \%$ at $313 \mathrm{~nm}$ excitation. ${ }^{\mathbf{1 9 , 2 0}}$ The formula used for QY measurement is as following:

$$
(\mathrm{QY})_{\mathrm{sm}}=(\mathrm{QY})_{\mathrm{st}} \frac{(\mathrm{FA} / \mathrm{Ab})_{\mathrm{sm}}}{(\mathrm{FA} / \mathrm{Ab})_{\mathrm{st}}} \frac{\left(\eta_{\mathrm{sm}}\right)^{2}}{\left(\eta_{\mathrm{st}}\right)^{2}}
$$

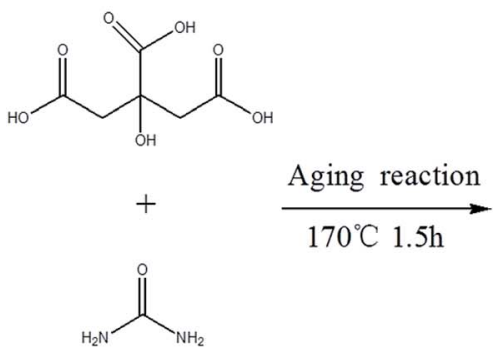

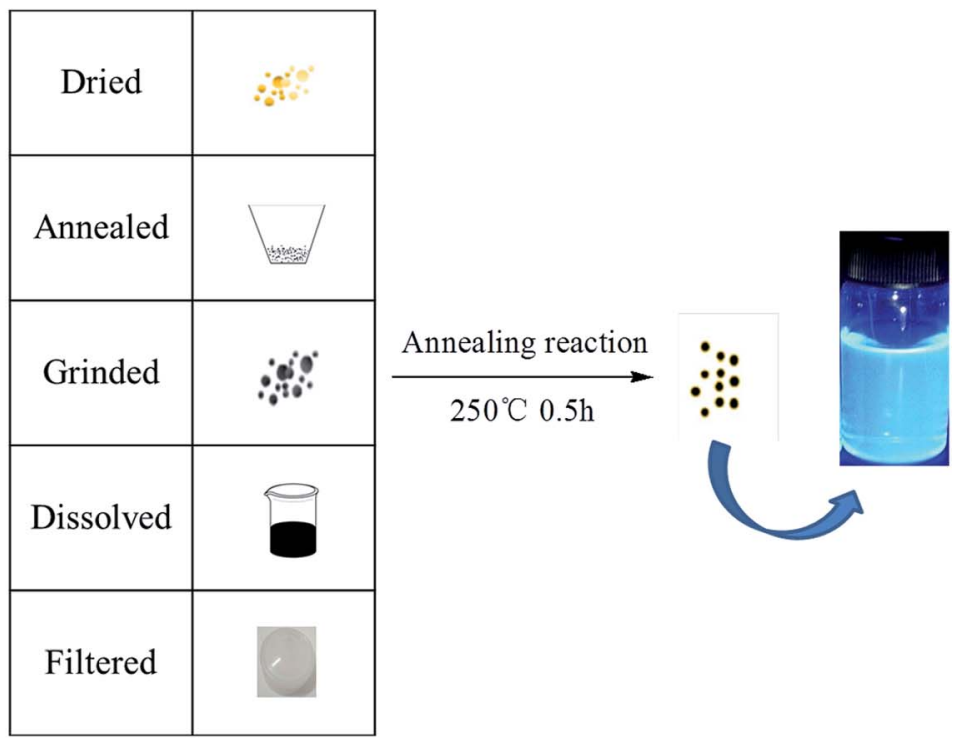

Scheme 1 Synthesis process of the CA-N-FCDs. 
where sm represents the sample, st represents the standard substance, $\eta$ indicates the refractive index of the solvent, FA represents the fluorescent integral area, and $\mathrm{Ab}$ indicates the absorbance value.

\subsection{Cytotoxicity assay}

Cytotoxicity measurement is based on MTT assay method. ${ }^{21}$ HeLa cells were incubated for $24 \mathrm{~h}$ with different dosages of the CA-N-FCDs suspension in 96-well plates. Then, the cells were washed with PBS solution and the cell morphology was observed by a phase difference microscope. Next, $200 \mu \mathrm{L}$ of a newly prepared MTT solution ( $20 \mu \mathrm{L}$ MTT and $180 \mu \mathrm{L}$ DMEM) was added into each well and incubated for $4 \mathrm{~h}$. After the culture medium was poured out, the $150 \mu \mathrm{L}$ DMSO was added to each well and the absorbance was respectively measured at $490 \mathrm{~nm}$ by the use of a microplate reader. The amount of MTT crystals formed was proportional to the number of living cells in a certain range of cell population, assuming that the control sample without any FCDs embraced $100 \%$ viability.

\subsection{In vitro cellular imaging experiments}

HeLa cells were cultured in DMEM with $10.0 \%$ fetal bovine serum and $1.0 \%$ penicillin-streptomycin double antibodies in the atmosphere of $5.0 \% \mathrm{CO}_{2}$ at $37{ }^{\circ} \mathrm{C}$. In a chamber slide, the cells were mixed with $500 \mu \mathrm{L}$ of the above medium and $200 \mu \mathrm{L}$ of

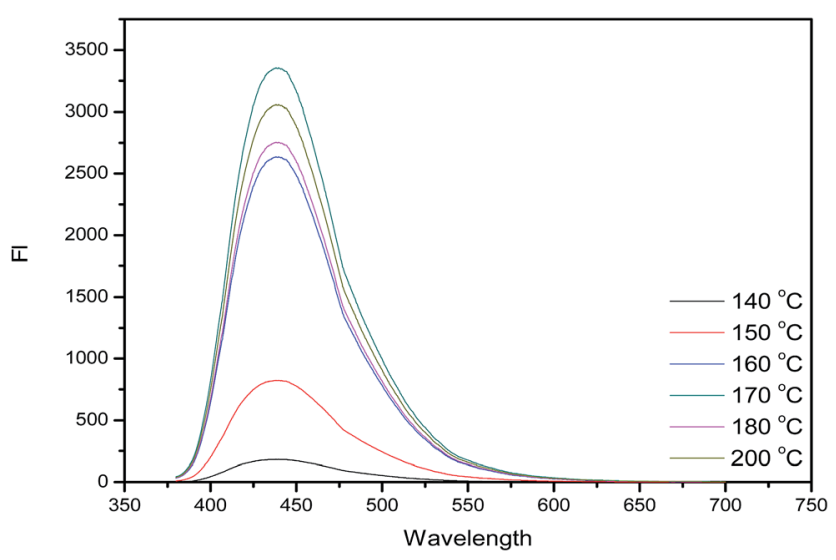

Fig. 1 Effect of the hydrothermal aging temperature on the fluorescence intensity of the CA-N-FCDs obtained by aging for $1.5 \mathrm{~h}$. the FCDS solution, and then incubated for $2 \mathrm{~h}$. After that, the incubation cells were washed with phosphate-buffered saline (PBS), fixed with $4.0 \%$ paraformaldehyde, mounted using $50.0 \%$ glycerol, and used for microscopic imaging. ${ }^{22-24}$

\subsection{Instrumentation}

Optical properties were performed using a V-530 UV-vis spectrophotometer and an F-4500 fluorescence spectrophotometer. Samples for the measurement of transmission electron microscopy (TEM) were prepared by putting a drop of particle suspension on a carbon-coated copper grid and observed with a JEM-2100 microscope. X-ray diffraction (XRD) was performed using a D/max-2550 PC X-ray powder diffractometer. Fourier transform infrared (FT-IR) spectroscopy was performed on an Avatar-380 FTIR spectrometer with solid KBr pellets. The fluorescence images of the cells were captured by DP72 microscope digital camera.

\section{Results and discussion}

\subsection{Effects of hydrothermal aging conditions}

3.1.1 Influence of aging temperature. The hydrothermal aging temperature shown in Fig. 1 and Table 1 was altered from $140{ }^{\circ} \mathrm{C}$ to $200{ }^{\circ} \mathrm{C}$, inclusive of $153{ }^{\circ} \mathrm{C}$, the reported melting point of CA. The aging time was $1.5 \mathrm{~h}$ under the adding ratio of $1: 1$ of $\mathrm{CA}$ to $\mathrm{N}$ in weight. The CA was barely carbonized below the melting point and the brightest sample was produced at $170{ }^{\circ} \mathrm{C}$ under the excitation of $365 \mathrm{~nm}$ light. When the temperature reached $180{ }^{\circ} \mathrm{C}$, the fluorescence intensity of the product became lower probably due to be over carbonized. Interestingly, while the temperature rose to $200{ }^{\circ} \mathrm{C}$, the sample under the excitation of $365 \mathrm{~nm}$ light got brighter than that at $180{ }^{\circ} \mathrm{C}$, which can be resulted from the additional annealing. ${ }^{25}$

3.1.2 Influences of adding ratio in weight and adding amount of the raw materials. The temperature $170{ }^{\circ} \mathrm{C}$ has been proven to be the most suitable and the effect of adding ratio in weight and adding amount was investigated by $1.5 \mathrm{~h}$ of aging on the product based on this temperature. Several parallel tests were carried out to check the influence of adding ratio in weight and adding amount of the raw materials. Table 2 and Fig. 2 displayed the corresponding results. From Table 2 and Fig. 2, the fluorescence intensity of the products varied with different raw materials ratio. When any kind of the raw materials is not

Table 1 Effect of aging temperature on the fluorescence of the product

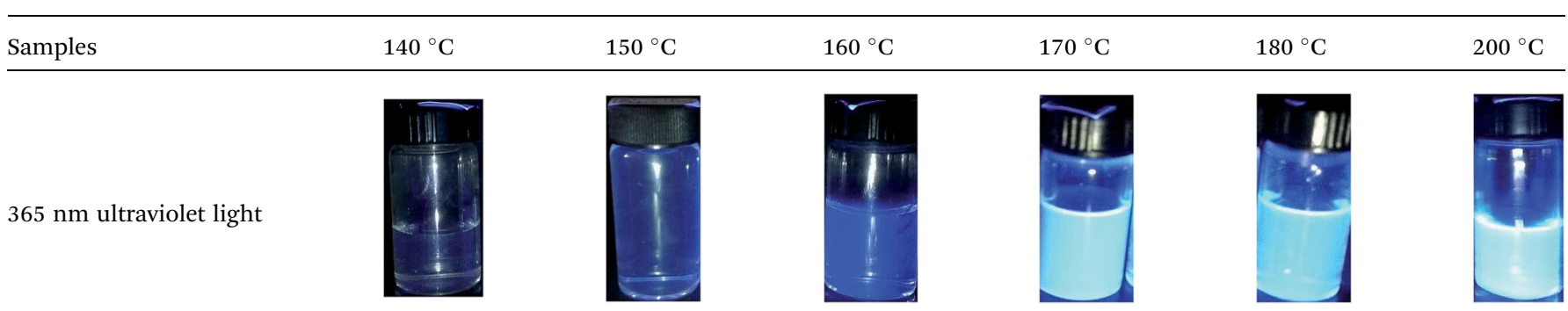


Table 2 Effect of adding ratio in weight on the fluorescence intensity of the product obtained at $170{ }^{\circ} \mathrm{C}$

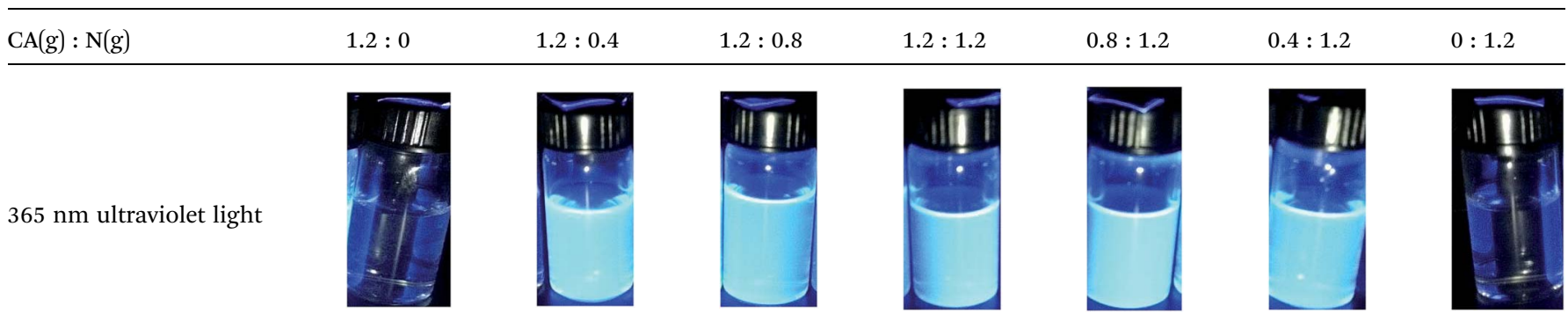

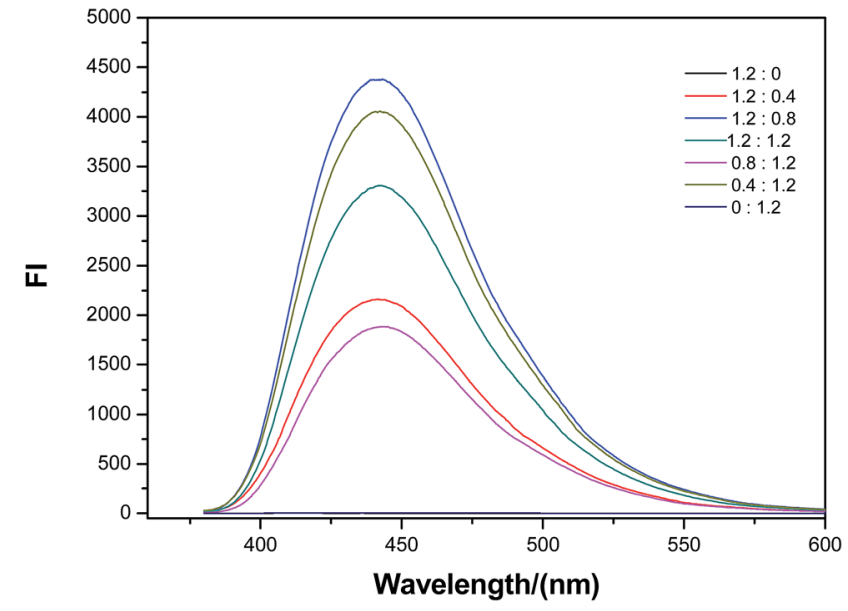

Fig. 2 Effect of the addition ratio in weight on the fluorescence intensity of the CA-N-FCDs obtained by aging at $170{ }^{\circ} \mathrm{C}$ (EM is the emission wavelength of the strongest fluorescence; $\mathrm{Fl}$ is the corresponding fluorescence intensity).

available, the strongly fluorescing product cannot be obtained. While the weight ratio of citric acid and urea is $1.2: 0.8$ in weight, the fluorescence intensity was the largest. The influence of adding amount of the raw material under the fixed weight ratio was further studied on the fluorescence properties. As shown in Table 3 [also see ESI, Table S1†] the fluorescence efficiency is the largest when citric acid amount is $1.2 \mathrm{~g}$, urea content is $0.8 \mathrm{~g}$. The yield of the product can reach $38.7 \%$, that is to say, the utilization ratio of $\mathrm{C}$ and $\mathrm{N}$ can reach $87.1 \%$, which provides the possibility for large-scale industrial production. By the use of $1.8 \mathrm{~g}$ citric acid and $1.2 \mathrm{~g}$ urea, UV absorbance is the strongest and the absorption band becomes wider [see ESI, Fig. S1†]. Therefore, $\mathrm{X} 5$ and $\mathrm{X} 7$ in Table 3 are more suitable for biological imaging and light conversion respectively. In a word, we established successfully a novel strategy or way to make the product embrace industrial probability, with a high scale yield.

3.1.3 Influence of aging time. The effect of aging time was investigated on the product based on the optimization conditions mentioned above. The results were exhibited in Fig. 3. Obviously, the largest intensity at $455 \mathrm{~nm}$ was found during $1.5 \mathrm{~h}$ from the right upper corner illustration of Fig. 3, in good agreement with the former fluorescence observation [see ESI, Table S2 $\dagger$ ]. When the aging lasted longer than $1.5 \mathrm{~h}$, the fluorescence intensity of the product became weaker probably caused by over carbonizing. In addition, when the aging time was over $2.5 \mathrm{~h}$, the intensity began to increase. This might be due to the recrystallization of the product and/or the promotion of the dispersity of the product. Interestingly, the influences from the time and the former temperature in Section 3.1.1 on the carbonization and recrystallization are similar.

\subsection{Effect of annealing condition}

The QY of the products obtained by optimization conditions of the aging method is only $51.7 \%$ and carbon spots were found with serious accumulation. In common, it is possible to promote the QY and dispersity of the products by annealing or calcination. So, the products from the aging were tried to be annealed for making the product more perfect. The fluorescence properties of the CA-N-FCDs changed under different annealing temperature and the optimized aging conditions were described in Fig. 4. The product after annealing had a low brightness obtained under too low or too high temperature. The brightest sample was produced by annealing at $250{ }^{\circ} \mathrm{C}$ under the excitation of $365 \mathrm{~nm}$ light. The QY after annealing is $78.8 \%$, which is $27.1 \%$ larger than that before annealing. In other words, the QY increased by more than 50\%. In Fig. 5, the XRD profiles at different annealing temperatures were displayed by comparison to the graphite standard pdf cards inserted in it. It

Table 3 UV absorbance and fluorescence intensity at different adding amounts with the same addition ratio

\begin{tabular}{|c|c|c|c|c|c|c|c|}
\hline Samples & $\mathrm{X}_{1}$ & $\mathrm{X}_{2}$ & $\mathrm{X}_{3}$ & $\mathrm{X}_{4}$ & $\mathrm{X}_{5}$ & $\mathrm{X}_{6}$ & $\mathrm{X}_{7}$ \\
\hline $\mathrm{CA}(\mathrm{g})+\mathrm{N}(\mathrm{g})$ & $0.15+0.1$ & $0.3+0.2$ & $0.6+0.4$ & $0.9+0.6$ & $1.2+0.8$ & $1.46+1.0$ & $1.8+1.2$ \\
\hline Fluorescence intensity $(a)$ & 33.61 & 256.8 & 1664 & 6127 & 9947 & 3948 & 8030 \\
\hline Fluorescence efficiency $(\gamma)$ & 88.19 & 875.8 & 5384.9 & 11633.7 & 20732.4 & 10855.1 & 6145.5 \\
\hline
\end{tabular}




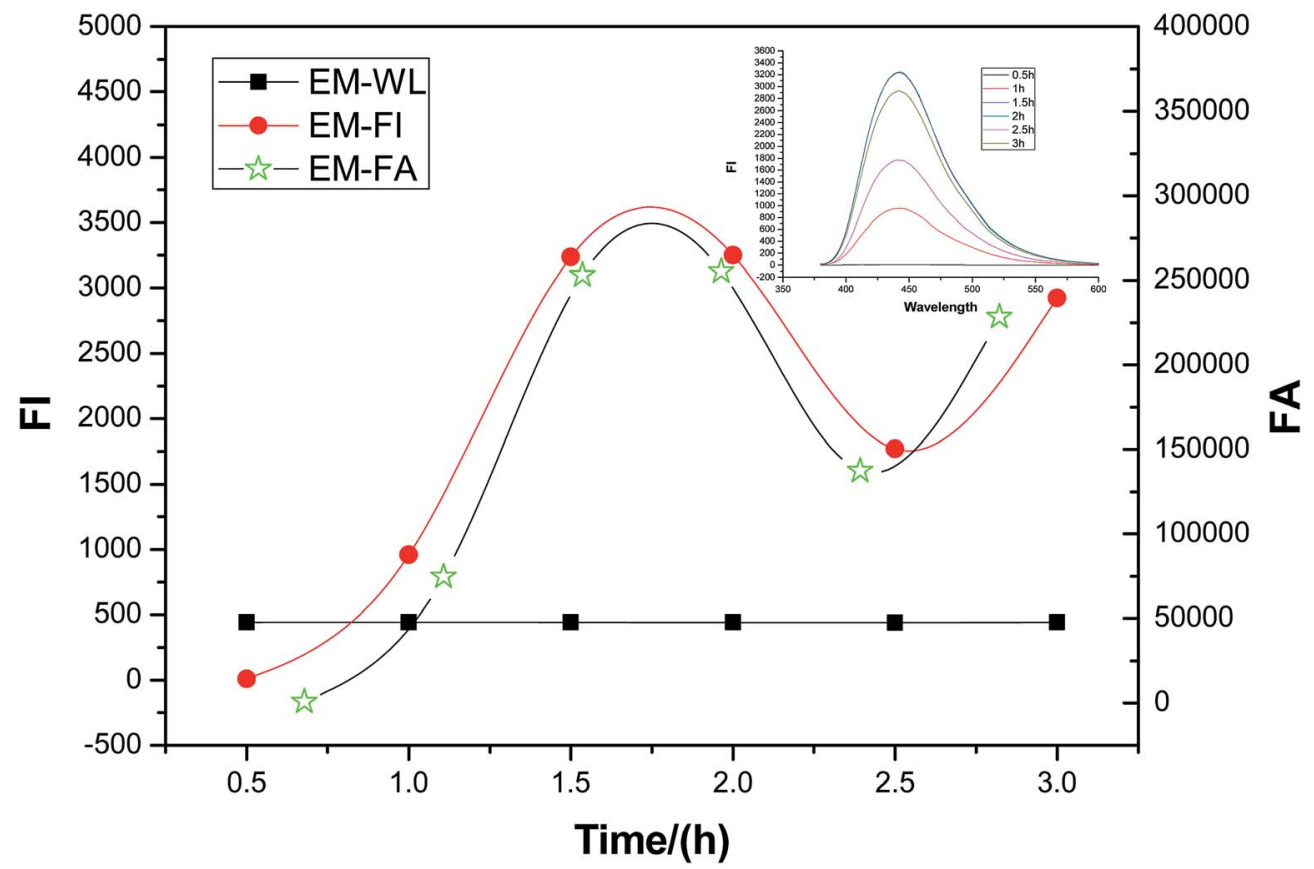

Fig. 3 Effect of the time on the fluorescence intensity of the $\mathrm{CA}-\mathrm{N}-\mathrm{FCD}$ s obtained by aging at $170{ }^{\circ} \mathrm{C}$ (the right upper corner illustration is the fluorescence spectra under excitation of $365 \mathrm{~nm}$; EM-WL is the emission wavelength of the strongest fluorescence; EM-FI is the corresponding fluorescence intensity; EM-FA is the fluorescence peak integral area).

was concluded that at other temperatures, the sample was partially graphitized and contained much disordered carbon structure, resulting in a weaker emission. However, the CA-NFCDs were close to perfect graphite crystals at annealing temperature of $250{ }^{\circ} \mathrm{C}$, in good agreement with the fact of the strongest emission. ${ }^{26}$
Consequently, we optimized the conditions for producing the high quality CA-N-FCDs in a large quantity. The hydrothermal temperature should be around $170{ }^{\circ} \mathrm{C}$ and duration time should be $1.5 \mathrm{~h}$. The adding weight ratio of citric acid and urea is $1.2: 0.8$. We further measured adding amounts under certain volume. The fluorescence efficiency is the largest when

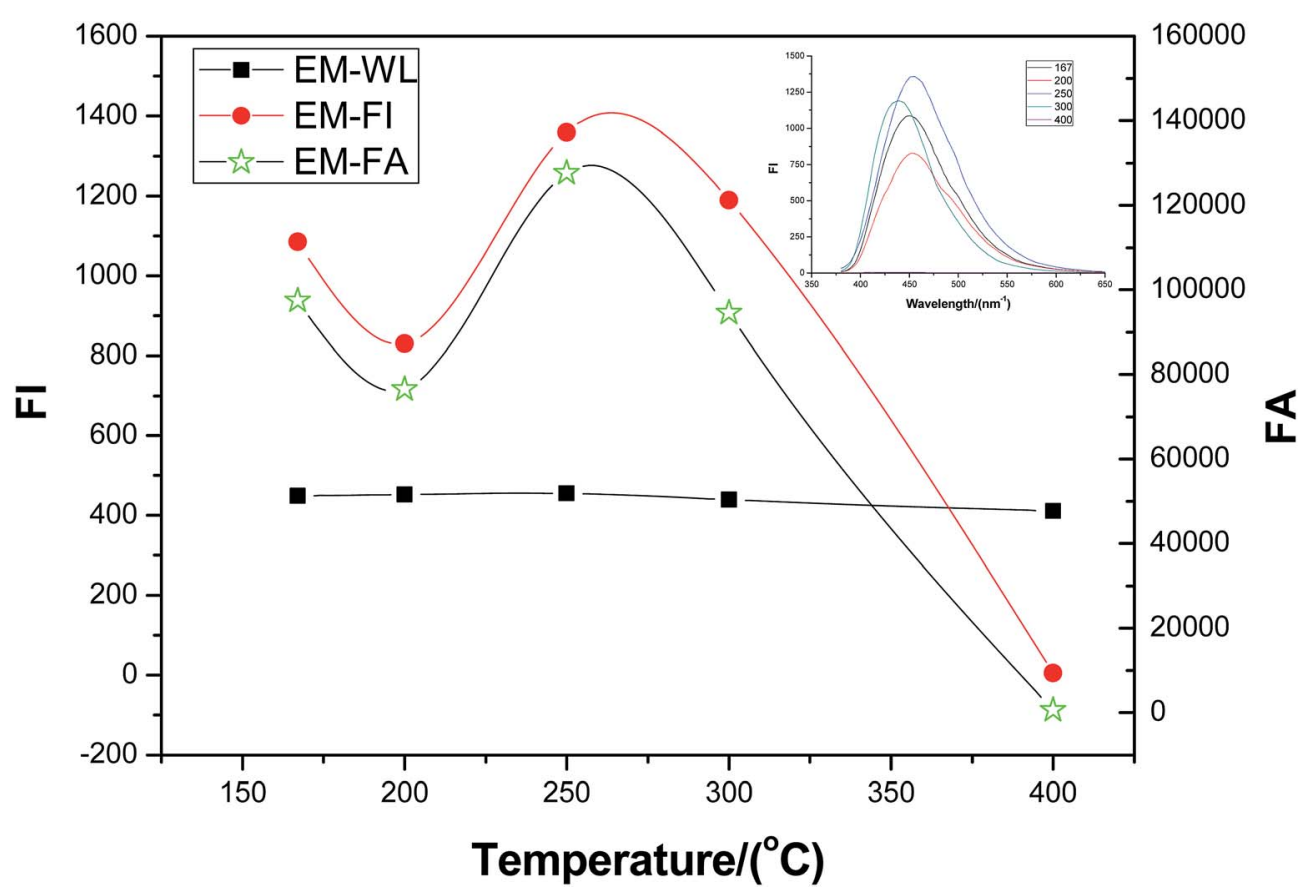

Fig. 4 Effect of the annealing temperature on the fluorescence intensity of the CA-N-FCDs obtained by aging at $170{ }^{\circ} \mathrm{C}$ (the right upper corner illustration is fluorescence spectra under excitation of $365 \mathrm{~nm}$; EM-WL is the emission wavelength of the strongest fluorescence; EM-FI is the corresponding fluorescence intensity; EM-FA is the fluorescence peak integral area). 


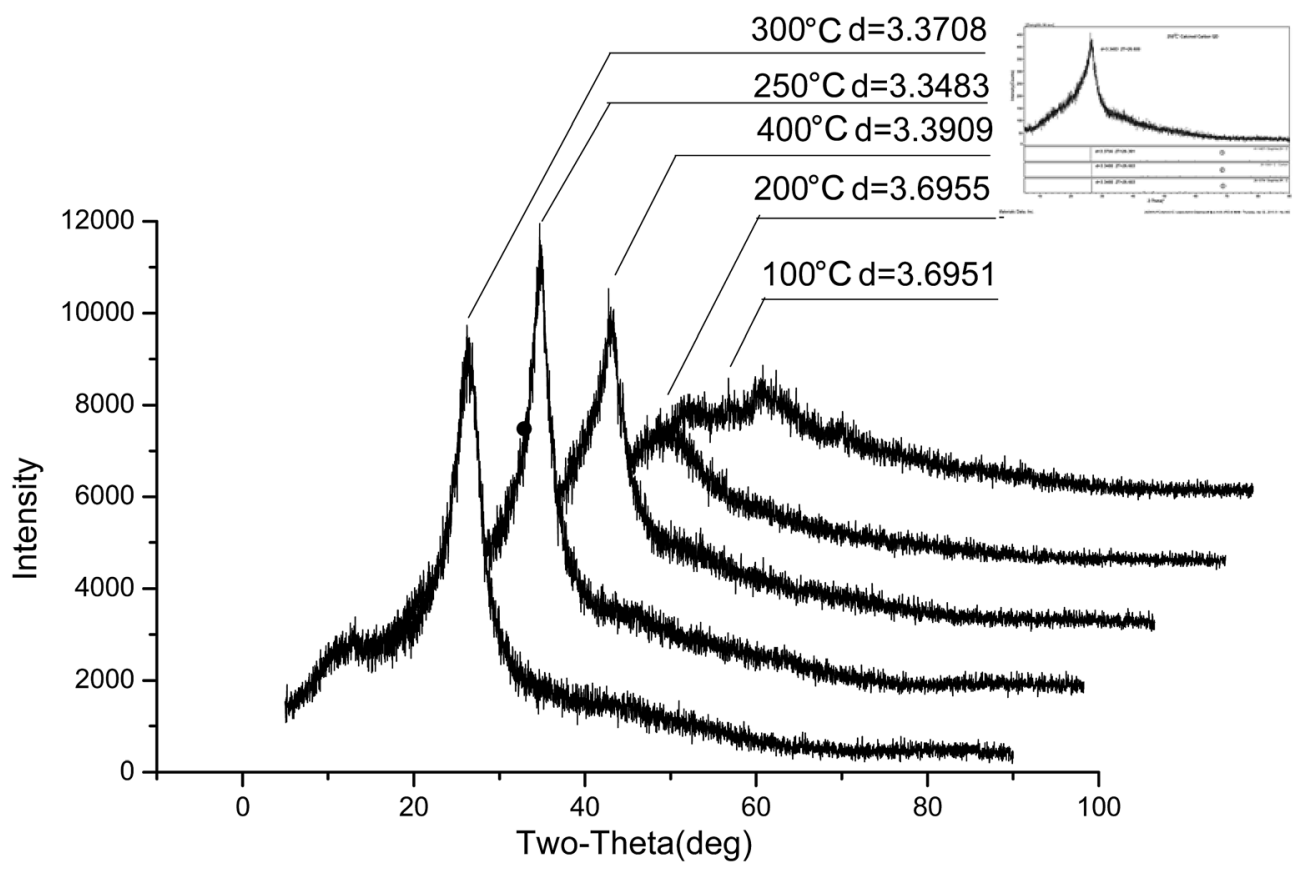

Fig. 5 XRD patterns of the CA-N-FCDs produced under different annealing temperature (the right upper corner illustration is the standard graphite pdf cards).

citric acid amount is $1.2 \mathrm{~g}$, urea content is $0.8 \mathrm{~g}$ in $25 \mathrm{~mL}$ of the distilled water. The fluorescence of the product became worse when using the single hydrothermal treatment at $170{ }^{\circ} \mathrm{C}$ or $250{ }^{\circ} \mathrm{C}$, in comparison to the current combined processing. After the product was dried, the utilization ratio of $\mathrm{C}$ and $\mathrm{N}$ reached $87.1 \%$, which promoted the possibility for industrial production. Meanwhile, when the annealing temperature is $250{ }^{\circ} \mathrm{C}$, not only the fluorescence intensity is optimum, but also it is mostly close to the graphite crystal type. Also, when only using the single annealing at $170{ }^{\circ} \mathrm{C}$ or $250{ }^{\circ} \mathrm{C}$, the process was not controllable, resulting in quite wide size-distribution of the formed CDs.

\subsection{Characterization of FCDs}

3.3.1 Effect of $\mathrm{pH}$ on the fluorescence intensity of the CA$\mathbf{N}-\mathbf{F C D}$. The CA-N-FCDs powder was dispersed in water with

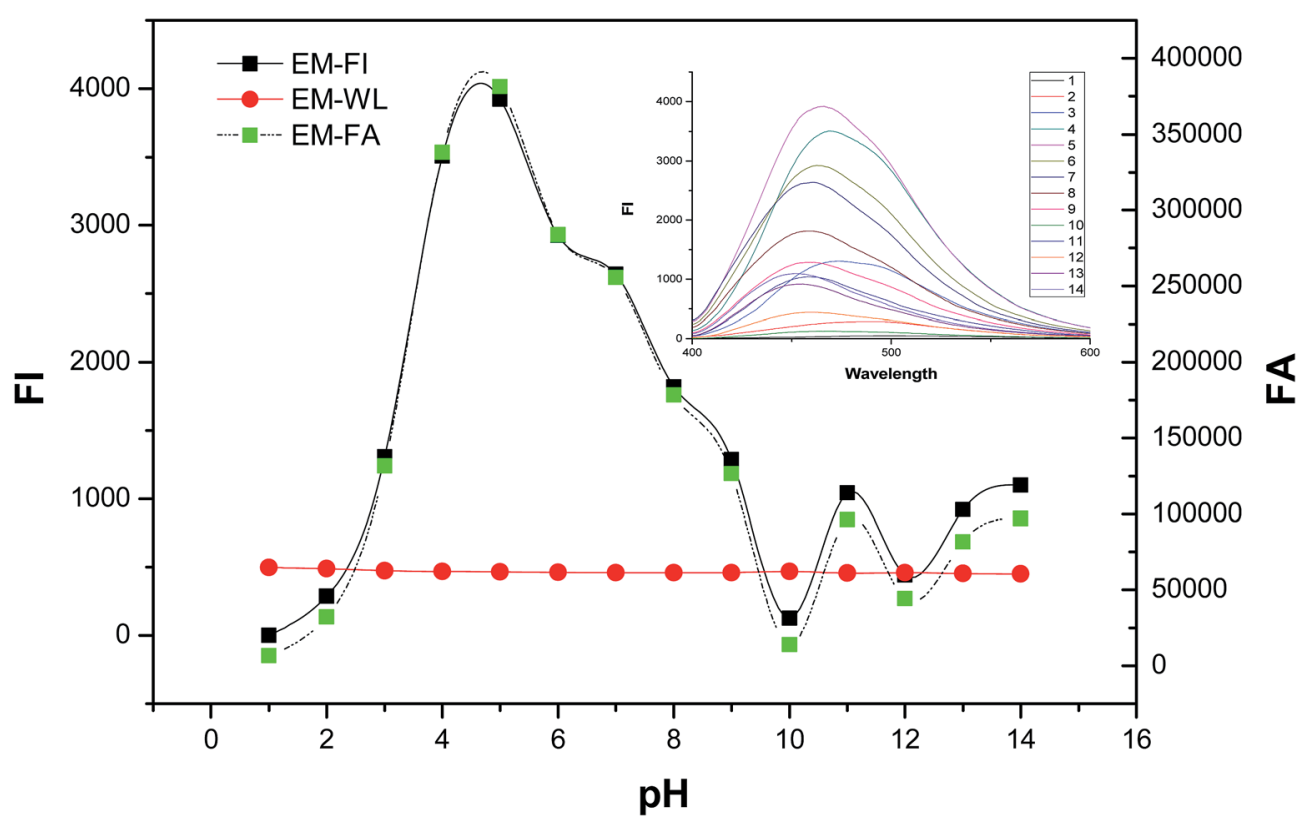

Fig. 6 Effect of the environmental $\mathrm{pH}$ on the fluorescence intensity of the CA-N-FCDs obtained at the optimal conditions (the right upper corner illustration is fluorescence spectra under excitation of $365 \mathrm{~nm}$; EM-WL is the emission wavelength of the strongest fluorescence; $\mathrm{EM}$-Fl is the corresponding fluorescence intensity; EM-FA is the fluorescence peak integral are). 


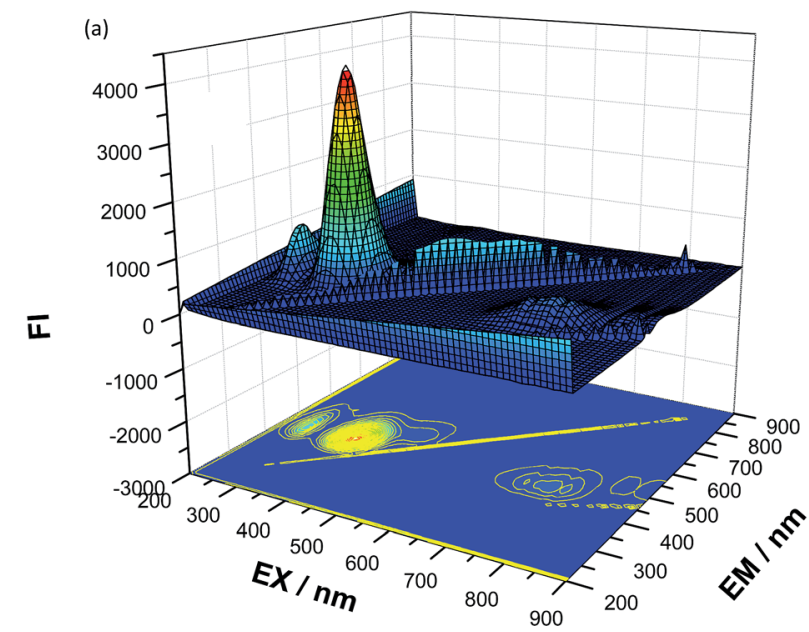

(b)

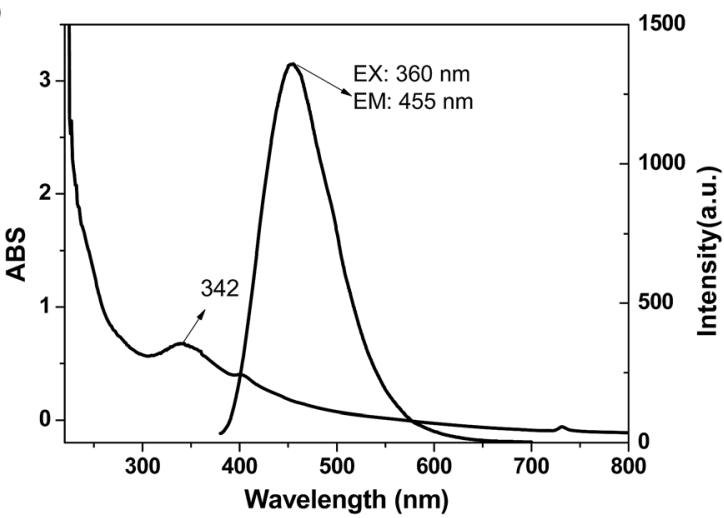

Fig. 7 Basic properties of the CA-N-FCDs: (a) 3D scanning map of fluorescence from the FCDs aqueous suspension, the image on the bottom is the contour map; (b) basic UV-vis absorption and fluorescence spectra. different $\mathrm{pH}$ adjusted with diluted $\mathrm{NaOH}$ or $\mathrm{HCl}$ solution, to study the $\mathrm{pH}$ influence. Fig. 6 shows $\mathrm{pH}$ effect on the fluorescence intensity on the CA-N-FCDs. It is found that the fluorescence emission peak wavelength remains almost the same at different $\mathrm{pH}$ values, indicating an excellent stability for the emission wavelength of the FCDs at different $\mathrm{pH}$ values, whereas, the largest fluorescence intensity is found to be at around $\mathrm{pH}$ 5.0. The $\mathrm{CA}-\mathrm{N}-\mathrm{FCD}$ are sensitive to the acidic environment, suggesting a possibility to be a novel $\mathrm{pH}$ probe, which may facilitate localization of tumor tissues.

3.3.2 Optical properties of the CA-N-FCDs. The optical properties of the $\mathrm{CA}-\mathrm{N}-\mathrm{FCDS}$ are shown in Fig. 7 under different excitation and emission wavelengths. Under different excitation wavelengths, the fluorescence spectra of the CA-NFCDs were measured to display the corresponding emission wavelengths (Fig. 7a). As a result, the best excitation wavelength is $365 \mathrm{~nm}$ which belongs to the near ultraviolet region and the corresponding emission is at $455 \mathrm{~nm}$ (Fig. 7b). As described in Fig. $7 \mathrm{~b}$, the carbon dots aqueous solution has a strong ultraviolet absorption at $342 \mathrm{~nm}$ and a large Stokes-shift more than $100 \mathrm{~nm}$. The phenomenon is commonly reported, possibly due to the band-gap renormalization effect. ${ }^{27}$ We recognize intuitively from Fig. 1a that the emission wavelength always focuses around $455 \mathrm{~nm}$ when the excitation wavelength changes. There are two main regions on the contour map (Fig. 7a), which respectively represent the up and down conversion properties of CA-N-FCDs. Virtually, the latter is more efficient. ${ }^{22}$

3.3.3 Morphology of the CN-N-FCDs. The size and morphology of the carbon dots were examined by using transmission electron microscope (TEM) and dynamic light scattering (DLS), and the results were shown in Fig. 8. From Fig. 6a, the well dispersed CA-N-FCDs are almost irregular shaped like a rectangle with a size ranged in $\mathbf{3 . 0 - 1 1 . 0 ~} \mathrm{nm}$. The average size of the produced CA-N-FCDs is $6.6 \mathrm{~nm}$ counted from TEM images. There is a normal difference between the data from TEM images and the hydrodynamic radius. The HRTEM image
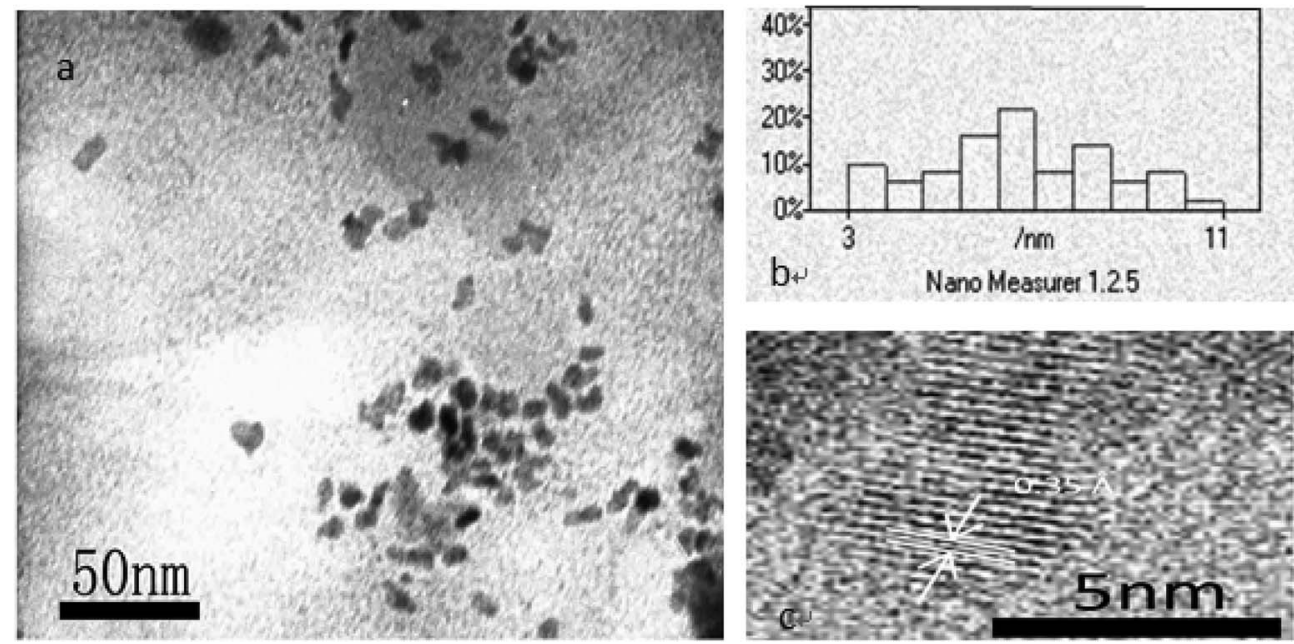

Fig. 8 Morphology characteristics of the CA-N-FCDs: (a) TEM of these FCDs; (b) the particle size statistical chart of FCDs; (c) HRTEM of the FCDs showing the lattice pattern of these crystals. 
shown in Fig. 8c suggests carbonization of the CA-N-FCDs. The lattice of the CA-N-FCDs is $3.35 \AA$ that is completely close to the graphite lattice (3.34 $\AA$ ), in good agreement with the XRD date as well. In other words, the final products are close to perfect graphite crystals. Besides $\mathrm{sp}^{2}$ hybridization, there is a small part of the $\mathrm{sp}^{3}$ hybridization, leading to $c$ non-perfect graphite lattice.

3.3.4 Cytotoxicity of the CA-N-FCDs. The compatibility between cells and the CA-N-FCDs is of vital importance to apply them to bio-imaging. Since the prepared CA-N-FCDs had excellent optical properties such as high fluorescence intensity and stability, the cytotoxicity of these FCDs was necessarily investigated through conventional MTT assay and phase difference analysis. Images of the phase difference analysis shown in Fig. 9a-d indicates that the cells are all spindleshaped in PBS and in the samples with the CA-N-FCDs of different concentrations, suggesting that the CA-N-FCDs do not affect the cell morphology even with a concentration as high as $678 \mu \mathrm{g} \mathrm{mL} \mathrm{m}^{-1}$. Additionally, the cell viability is also more than $90 \%$ with the concentration of CA-N-FCDs more than $600 \mu \mathrm{g}$ $\mathrm{mL}^{-1}$, as illustrated in Fig. 10. In contrast, it is known that CdSe-based QDs $^{28}$ and rhodamine ${ }^{29}$ are strongly cytotoxic in such a high dosage. That is to say, the CA-N-FCDs have extremely low cell cytotoxicity for being a kind of safe biomaterials. However, it is known that, the cells with any substance in them can be damaged if its concentration is too high. In the current case, the cells were busted in the presence of the CA-N-FCDs with a concentration as high as $10 \mathrm{mg} \mathrm{mL}^{-1}$.

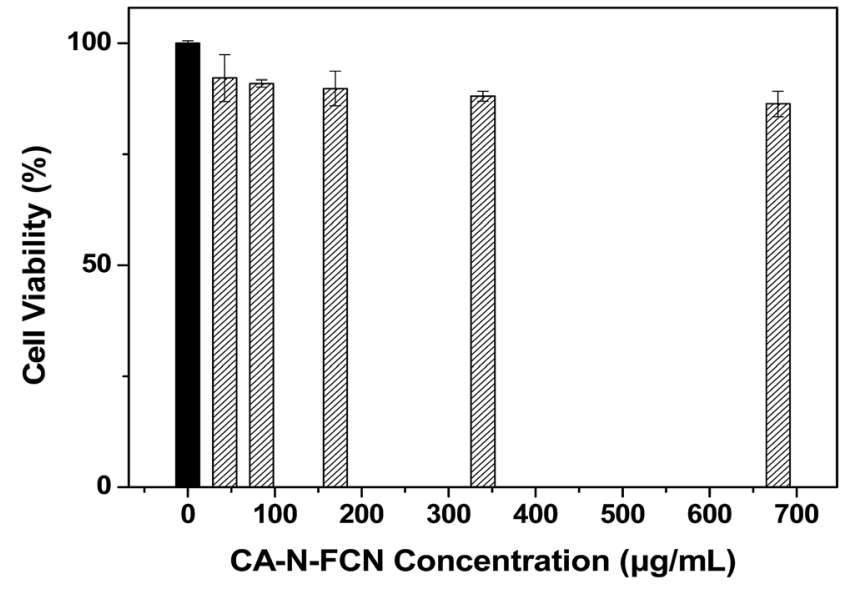

Fig. 10 The cell viability obtained by MTT assay.

\subsection{Application as a bio-imaging probe}

Colloidal stability for the CA-N-FCDs was tested under different aqueous buffer solutions. The results showed that the solutions were very stable within months, indicating that the material can be ideally used as a bio-probe. Besides, the low cell cytotoxicity also determines them to be an outstanding bio-probe.

Nevertheless, it is quite important to apply the CA-N-FCDs to be a fluorescent probe in vitro. HeLa cells were incubated with the CA-N-FCDs dissolved in PBS for $2 \mathrm{~h}$ in order to make the materials enter the cells completely. Then, the washed cells were fixed with $4.0 \%$ paraformaldehyde solution and observed

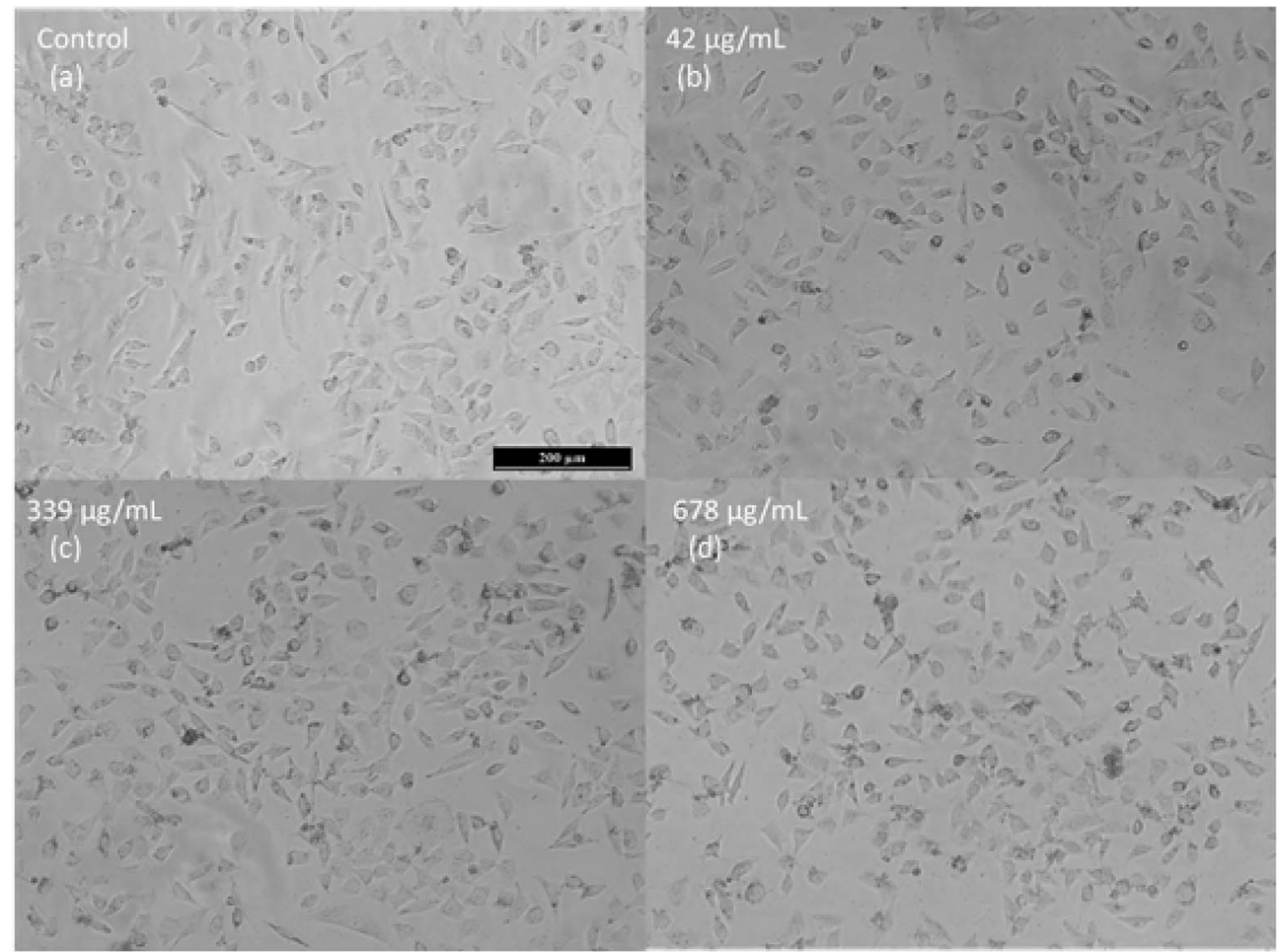

Fig. 9 Phase contrast images of the CA-N-FCDs to show the cytotoxicity: (a) cells added only with PBS; (b) cells added with $42 \mu \mathrm{g} \mathrm{mL}^{-1} \mathrm{CA}-\mathrm{N}-$ FCDs solution; (c) cells added with $210 \mu \mathrm{g} \mathrm{mL}{ }^{-1} \mathrm{CA}-\mathrm{N}-\mathrm{FCDs}$ solution; (d) cells added with $678 \mu \mathrm{g} \mathrm{mL} \mathrm{m}^{-1} \mathrm{CA}-\mathrm{N}-\mathrm{FCDs}$ solution. 


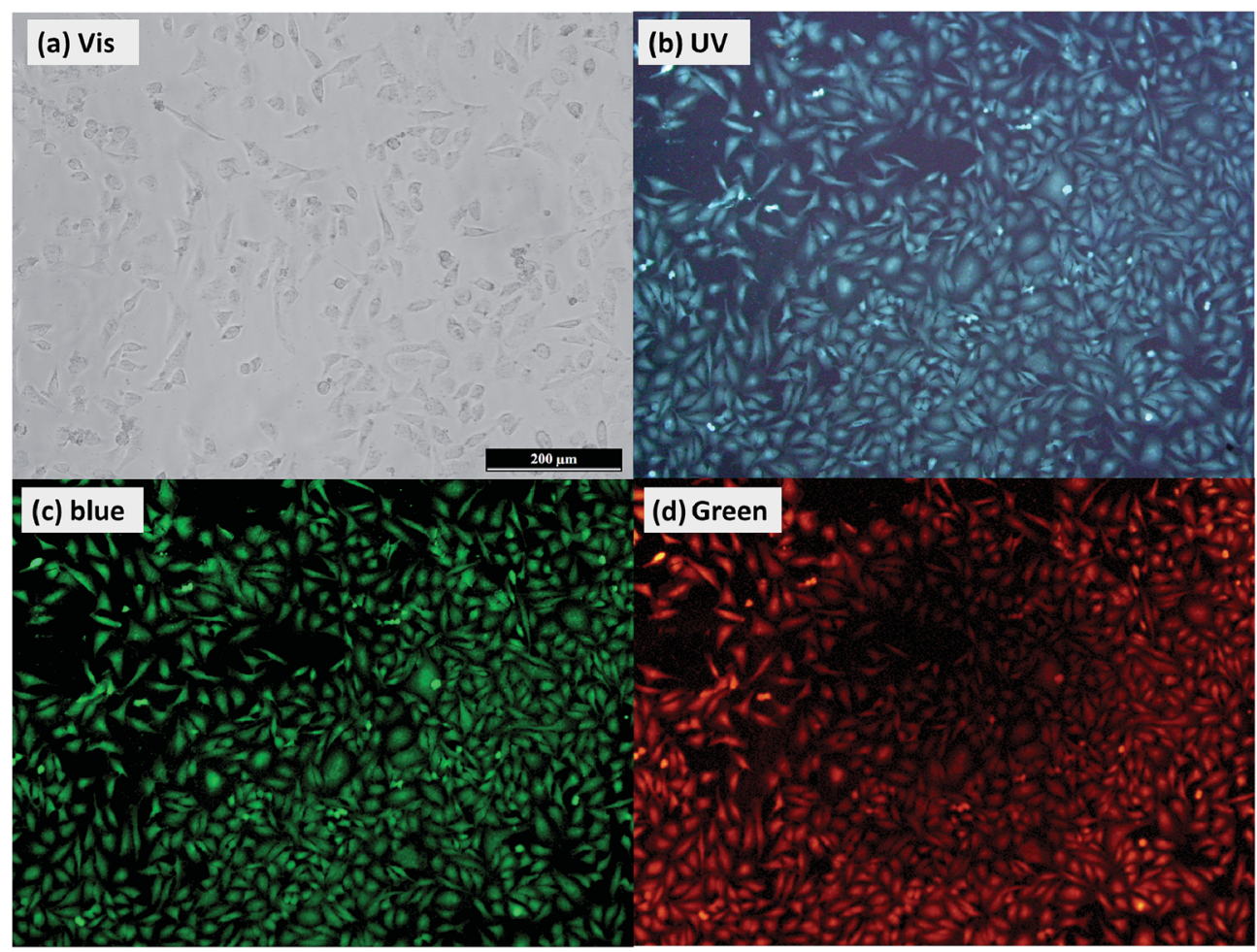

Fig. 11 Fluorescence images under excitation of different light sources ("Band Jump Phenomenon"): (a) the cells labeled with the FCDs under visible light excitation; (b) the labeled cells under UV co-excitation from 320 to $380 \mathrm{~nm}$; (c) the green emission labeled cells under composite blue light excitation from 465 to $490 \mathrm{~nm}$; (d) the red emission labeled cells under composite green light excitation from 530 to $570 \mathrm{~nm}$ (for interpretation of the references to color in this figure legend, the reader is referred to the web version of this article).

under a fluorescence microscope. As we all know, the color of cells without marker cannot be altered when excited by lights in a color band, so the control test is not needed. Fig. 11 describes the morphologies of those HeLa cells labeled by the CA-NFCDs. As the mercury lamp of fluorescence microscope used belongs to a linear light source, we found the most important feature of our product which is not easy to be observed in the former reports. It is called "Band Jump Phenomenon" (tricolor phenomenon). ${ }^{29}$ Fig. 11a shows the cell morphology labeled with the FCDs under visible light irradiation. The cells are blue under UV co-excitation from 320 to $380 \mathrm{~nm}$ as shown in Fig. 9b. Then, they become bright green under co-excitation of blue light from 465 to $490 \mathrm{~nm}$ and turn red under composite green excitation from 530 to $570 \mathrm{~nm}$ (Fig. 11c and d). In addition, Fig. 11 also shows the materials can rapidly enter into the cells. Moreover, we can see from these pictures that the morphological resolution of the labeled cell under any of the latter three bands is higher than that under visible light. Also, red and green images are more vivid and brighter than other colors under the same conditions. Further, we can even observe the details under special excitation bands, such as nucleus and cytoplasm by using so popular fluorescent microscope.

The synthesis conditions and process (Scheme 1) are very simple, green and safe as well. The hydrothermal aging temperature of the method is $170{ }^{\circ} \mathrm{C}$ and the annealing temperature is $250{ }^{\circ} \mathrm{C}$. The most meaningful thing is that the high QY of the FCDs. The resulting FCDs exhibit a stable fluorescence suitable for cell imaging application. Even more, they may be used as immune marker ${ }^{21}$ and in bio-imaging in vivo. ${ }^{22,23}$ Fortunately, our product can emit three different colors under three kinds of different excitation lights. From the previous reports, the red luminescent FCDs were difficult to be produced, ${ }^{22,24}$ but our product can emit three kinds of lights including red luminescence. Especial imaging with the red and green luminescence is more vivid than blue one. In a word, our strategy provides a uniform heating environment, suitable temperature, for achieving high fluorescence quantum efficiency of the FCDs with a high yield in a large quantity. Thus, an easier industrialization method to produce FCDs is founded, as the basis for a variety of practical applications.

\section{Conclusions}

In summary, high-quality CDs were successfully prepared by using citric acid (CA) and urea ( $\mathrm{N}$ source) as initial reactants, in which the hydrothermal aging and the consequential annealing were combined effectively. By adjusting the reaction parameters such as hydrothermal aging temperature, adding ratio, adding amount, duration time, the FCDs with high QY and minimal toxicity were used in animal cell fluorescence imaging. We establish a novel way to make the QY of FCDs is $78.8 \%$ which is the highest datum among all the known reports. Under the mild conditions, these raw materials derived FCDs display amused luminescent properties, including high stability, low cell 
cytotoxicity, and outstanding performance as a fluorescence probe. We exhibited a perfect performance under 3 kinds of excitation bands (UV, blue, and green lights). Anyway, this article discusses the characteristics of the FCDs in details and provides an easier industrialization method to produce FCDs, as the basis for a variety of applications liking imaging in vitro and vivo. ${ }^{31}$ Compared with using the single hydrothermal treatment or the annealing at relatively low temperature, the combination of the two methods can make the excellent CDs, that will greatly push the practical application. In a word, the CA-N-FCDs are a kind of perfect probes for bio-imaging and will attract more and more attentions in the near future.

\section{Conflicts of interest}

The authors declare no competing financial interest.

\section{Acknowledgements}

The authors are thankful for support from the fluorescence microscope instrument facility of Professor Hong research group and the technical supporting from Professor Shi research group in cell trials.

\section{References}

1 X. Y. Xu, R. Ray, Y. L. Gu, H. J. Ploehn, L. Gearheart, K. Raker and W. A. Scrivens, J. Am. Chem. Soc., 2004, 126, 1273612737.

2 G. Gedda, C. Y. Lee, Y. C. Lin and H. F. Wu, Sens. Actuators, B, 2016, 224, 396-403.

3 D. Bu, H. S. Zhuang, G. X. Yang and X. X. Ping, Sens. Actuators, B, 2014, 195, 540-548.

4 N. Na, T. T. Liu, S. H. Xu, Y. Zhang, D. C. He, L. Y. Huang and J. Ouyang, J. Mater. Chem. B, 2013, 1, 787-792.

5 L. M. Hu, Y. Sun, S. L. Li, X. L. Wang, K. L. Hu, L. R. Wang, X. J. Liang and Y. Wu, Carbon, 2014, 67, 508-513.

6 M. Yu, X. Z. Li, F. Zeng, F. Y. Zheng and S. Z. Wu, Chem. Commun., 2013, 49, 403-405.

7 R. H. Liu, H. Huang, H. T. Li, Y. Liu, J. Zhong, Y. Y. Li, S. Zhang and Z. H. Kang, ACS Catal., 2014, 4, 328-336.

8 C. W. Lai, Y. H. Hsiao, Y. K. Peng and P. T. Chou, J. Mater. Chem., 2012, 22, 14403-14409.

9 R. Yan, H. Wu, Q. Zheng, J. Y. Wang, J. L. Huang, K. J. Ding, Q. G. Guo and J. Z. Wang, RSC Adv., 2014, 4, 23097-23106.

10 J. Xu, S. Sahu, L. Cao, P. Anilkumar, K. N. Tackett II, H. J. Qian, C. E. Bunker, E. A. Guliants, A. Parenzan and Y. P. Sun, ChemPhysChem, 2015, 12, 3604-3608.
11 Q. L. Wang, H. Z. Zheng, Y. J. Long, L. Y. Zhang, M. Gao and W. J. Bai, Carbon, 2011, 49, 3134-3140.

12 H. Zhu, X. L. Wang, Y. L. Li, Z. J. Wang, F. Yang and X. R. Yang, Chem. Commun., 2009, 1, 5118-5120.

13 H. Y. Wu, C. C. Mi, H. Q. Huang, B. F. Han, J. Li and S. K. Xu, J. Lumin., 2012, 132, 1603-1607.

14 Z. C. Yang, X. Li and J. Wang, Carbon, 2011, 49, 5207-5212. 15 R. L. Liu, D. Q. Wu, S. H. Liu, K. Koynov, W. Knoll and Q. Li, Angew. Chem., Int. Ed., 2009, 48, 4598-4601.

16 H. Li, F. Q. Shao, S. Y. Zou, Q. J. Yang, H. Huang, J. J. Feng and A. J. Wang, Microchim. Acta, 2016, 183, 821-826.

17 Z. M. Zhang, Y. Pan, Y. N. Fang, L. L. Zhang, J. Y. Chen and C. Q. Yi, Nanoscale, 2016, 8, 500-507.

18 Q. L. Wang, H. Z. Zheng, Y. J. Long, L. Y. Zhang, M. Gao and W. J. Bai, Carbon, 2011, 49, 3134-3140.

19 B. X. Zhang, G. Y. Zhang, H. Gao, S. H. Wu, J. H. Chen and X. L. Li, RSC Adv., 2015, 5, 7395-7400.

20 X. Wang, L. Cao, S. T. Yang, F. Lu, M. J. Meziani, L. Tian, K. W. Sun, M. A. Bloodgood and Y. P. Sun, Angew. Chem., Int. Ed., 2010, 49, 5310-5314.

21 N. Gao, W. Yang, H. L. Nie, Y. Q. Gong, J. Jing, L. J. Gao and X. L. Zhang, Biosens. Bioelectron., 2017, 96, 300-307.

22 K. Jiang, S. Sun, L. Zhang, Y. h. Wang, C. Z. Cai and H. W. Lin, ACS Appl. Mater. Interfaces, 2015, 7, 23231-23238.

23 X. Yang, Y. H. Li, Y. Wang, J. Cui, X. Yin, X. He and Y. Zhang, Analyst, 2014, 139, 5134-5139.

24 Y. Huang, X. Zhou, R. Zhou, H. Zhang, K. Kang and M. Zhao, Chemistry, 2014, 20, 5640-5648.

25 J. H. Shem, Y. H. Zhu, C. Chen, X. L. Yang and C. Z. Li, Chem. Commun., 2011, 47, 2580-2582.

26 P. B. Sorokin, A. G. Kvashnin, Z. Zhu and D. Tománek, Nano Lett., 2014, 14, 7126-7130.

27 (a) S. A. O. Gomes, C. S. Vieira, D. B. Almeida, J. R. SantosMallet, R. F. Menna-Barreto, C. L. Cesar and D. Feder, Sensors, 2011, 11, 11664-11678; (b) J. H. Shem, Y. H. Zhu, C. Chen, X. L. Yang and C. Z. Li, Chem. Commun., 2011, 47, 2580-2582.

28 P. K. S. Magut, S. Das, V. E. Fernand, J. Losso, K. McDonough, B. M. Naylor, S. Aggarwal and I. M. Warner, J. Am. Chem. Soc., 2013, 135, 15873-15879.

29 N. N. Zheng, S. Ding and X. P. Zhou, Colloids Surf., B, 2016, 142, 123-129.

30 D. Zhao, C. Q. Zhao, M. Li, J. S. Ren and X. G. Qu, Anal. Chim. Acta, 2014, 809, 128-133.

31 Y. Huang, X. Zhou, R. Zhou, H. Zhang, K. Kang and M. Zhao, Chemistry, 2014, 20, 5640-5648. 